\title{
Regenerative Liquid Ring Pumps Review and Advances on Design and Performance
}

\author{
N.D. Karlsen-Davies, Dr G.A. Aggidis* \\ Lancaster University Renewable Energy and Fluid Machinery Group, Engineering Department, \\ Lancaster LA1 4YR, United Kingdom \\ *Corresponding author. \\ Email address: g.aggidis@lancaster.ac.uk (G.A. Aggidis)
}

\begin{abstract}
The regenerative liquid ring ( $R L R$ ) pump is a type of rotodynamic machine which has the ability to develop high heads at relatively low flow rates in only one impeller stage. Although the exact principle of operation of this type of pump has been a phenomenon not fully understood, it has nevertheless been widely applied for over a century in areas of liquid pumping. Despite the low efficiency, RLR pumps have several advantages over other turbomachines with similar tip speed due to relatively low manufacturing costs, simplicity, high reliability, enhanced priming behaviour and can in many applications offer a more efficient alternative. Efficiency improvements are key to reducing energy consumption and ultimately combatting the global climate change. This paper offers an extensive review into the development, performance challenges and design improvements of RLR pumps in order to provide some useful insight on future research and next steps, with a particular focus on improving efficiency throughout the pump life cycle.
\end{abstract}

\section{Keywords}

Regenerative liquid ring pumps, self-priming pumps, computational modelling, experimental modelling, performance challenges, design optimisation

\section{Introduction}

Pumps are the single largest user of electricity in the industry sector in the European Union [1]. A study on improving efficiency in pumps carried out by the European Commission in 2001 [1], found that pumps consumed as much as 160 TWh of electricity per year, equivalent to $14 \%$ of the total electricity consumed in industry and commerce. The energy wasted by those pumps was as high as 46 TWh in 2008 [2].

Centrifugal pumps represent some $73 \%$ of all pump energy consumption. The regenerative liquid ring pump (RLR) is, like the centrifugal pump, a kinetic machine. However it can in many applications offer a more efficient alternative [3]. Despite the many advantages over other turbomachines with similar tip speed, one of the main challenges of RLR pumps is to understand and improve the efficiency. In addition the degradation effects due to erosion, cavitation and natural wear life must be fully understood, as it is closely linked to pump efficiency. The rate of wear will tend to be greater during operation away from best-efficiency conditions. For example, larger clean water pumps can on average drop $5 \%$ in efficiency in the first five years of operation, partly due to wear [1]. It therefore becomes important not only to improve the hydraulic design of the RLR pump but also to assess efficiency-degrading parameters during its whole lifecycle. This review forms part of novel research into the life cycle assessment of a RLR pump using computational modelling techniques to improve its reliability.

The RLR pump is a type of rotodynamic pump which combines mechanical impulses of the impeller with centrifugal force [4]. The increase in head is achieved through a momentum exchange between 
the impeller and the pumped fluid [3]. However, in contrast to the centrifugal pump, the pressure rise occurs in the peripheral rather than in the radial direction [5-6]. Perhaps what really distinguishes the RLR pump is its ability to develop high heads at relatively low flow rates in only one impeller stage [7-8]. It shares similar operating characteristics to another pump classification, positive displacement: the power is directly proportional to head, with a maximum power required at shutoff and a very steep, nearly straight head-capacity curve [4, 9-11].

RLR pumps are also sometimes referred to as peripheral, side channel, water-ring, liquid-ring, drag, turbine, traction, tangential, vortex and claw-type pump in literature, often reflecting a variation in geometrical design or preference for a theory of the principle of operation [9, 12-13].

Figure 1 shows a schematic of a generic RLR pump. It consists of a radially split casing with an annular channel and an impeller [10] with several, generally 20-50 [7], radial blades around its perimeter. The annular channel is sometimes separated into the side channel, which are the channels at either side of the impeller, and the tip channel, which is the channel spanning all the way around the periphery of the impeller.

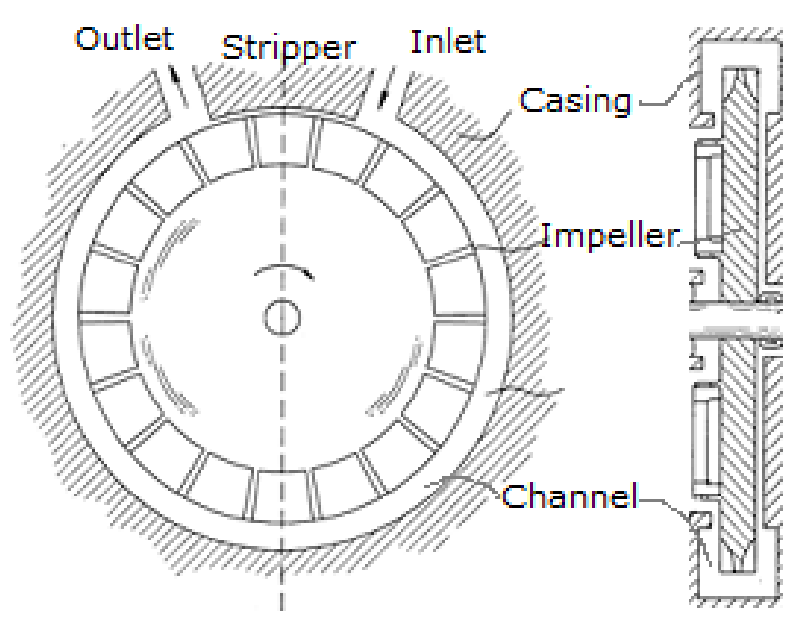

Figure 1: Schematic of a typical regenerative pump [14]

The inlet (suction) and outlet (discharge) ports are separated by a 'stripper region' with close clearance to the impeller, which prevents the fluid from flowing back from the outlet to the inlet, so only the fluid between the blades is allowed to pass through. The clearances between the impeller disk and the casing are kept to a minimum to prevent leakage from the high-pressure side back to the low-pressure side [12, 15-16].

The increase in head is achieved through an exchange of momentum between the impeller and the fluid. The fluid circulates through the blades as shown in in Figure 2. The fluid that enters into the lower blade space from the side channel in the direction parallel to the machine axis receives angular momentum by the rotating blades before it is radially discharged at the blade tip. The blade pocket fluid then transfers the angular momentum to the peripheral flow in the annular open channel, which is driven by shear force due to the rotating blades. The fluid that transfers all the momentum enters into the following blade space, again in an axial direction near the blade hub. This energy exchange process repeats itself from the inlet to the outlet ports, thus the static pressure continues to increase. Consequently, the fluid follows a helical passage throughout the flow channel (Figure 3). It is this repetitive motion of the fluid (regeneration) that allows these pumps to generate high heads at relatively low specific speeds in a single impeller stage. 


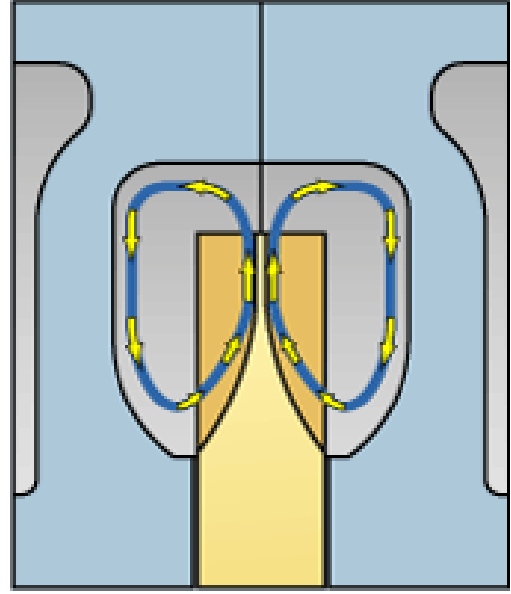

Figure 2: Channel cross-section showing flow circulation

[17]

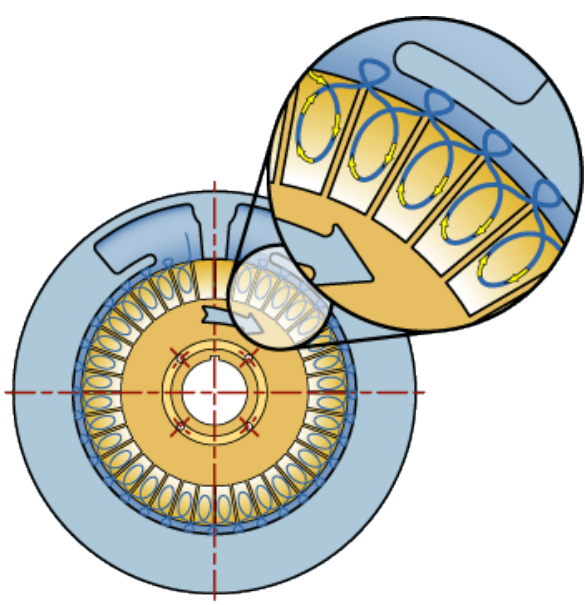

Figure 3: Casing cross section showing helical flow pattern [17]

\section{Background}

\subsection{History}

It is hard to pinpoint exactly when the RLR pump was invented due to its many names and forms. Perhaps one of the earliest concepts to make use of the regenerative operational principle in the United Kingdom was the helical pump by Imray [18]. It consisted of a paddle shaped wheel revolving within a cylindrical casing featuring fixed vanes, similar to parallel-flow turbines, which induced a helical flow pattern to the working fluid before it entered the wheel. Imray's helical pump [18] was later revived by Molloy [19] and Addison [20] in the form of a self-priming pump, although in Addison's version the impeller shaft was mounted eccentrically in the casing (similar to Figure 4). This was later found to be less efficient than a concentrically mounted impeller [21].

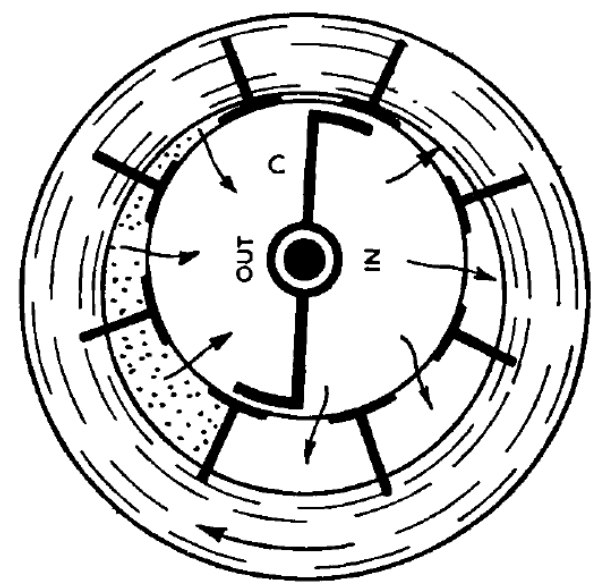

Figure 4: Diagram of Addison's liquid ring pump with an eccentrically mounted shaft [21]

The exact principle of operation of a regenerative pump has been a phenomenon that has intrigued many researchers through the years. It has been investigated using various theoretical models, experimental test studies and more recently computational modelling techniques.

The theoretical models can be a good tool for evaluating different geometry parameters quickly. However, they cannot capture the flow in a realistic manner due to simplified assumptions and the need for experimental correction data, which have a significant effect on the performance prediction. Promising experimental work in the 1940s and 50s [22-25], which made use of flow visualisation (Figure 5), was successful in determining the nature of the flow pattern but as with any experimental procedure, they can be time consuming and costly, not to mention the issue of capturing adequate detail. Computational modelling has showed good correlation with both 
theoretically and experimentally determined parameters. Although experimental testing is beneficial, the advanced computational tool is less costly and time consuming and offers high repeatability. However like all methods, it has its weaknesses. Often the geometry to be modelled has to be simplified due to limitations in processing capabilities, such as applying a coarse mesh in regions of detail, which may lead to inaccuracies [6].

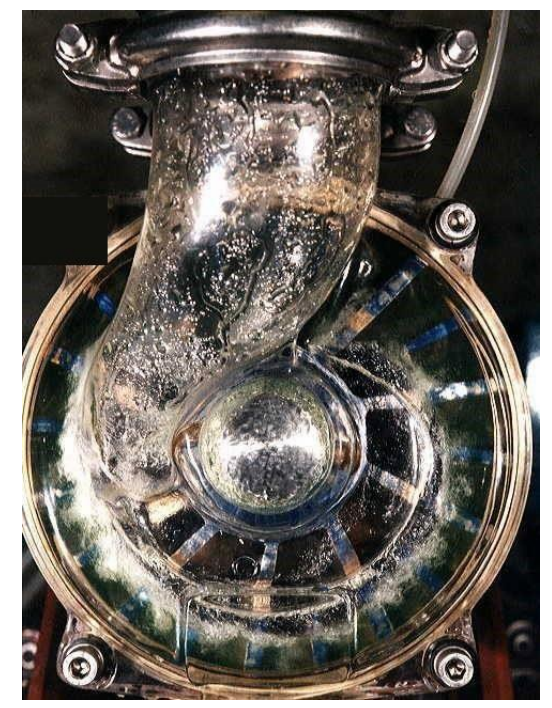

Figure 5: Flow visualisation study using Perspex glass [26]

There are two main theoretical models for describing the flow in regenerative pumps, each relying on a set of basic assumptions. These models can be categorised by the mechanism responsible for the flow pattern: turbulence or angular momentum exchange.

The turbulence mixing theory [27-28] considers a turbulence mechanism to be the main driving force of the flow. In some variations of this theory the turbulent stresses are transmitted by the mixing length theory, while in others turbulent shearing stresses are assumed between the impeller and fluid and between the fluid and casing. Flow visualisation studies conducted in the 1940s [22-23] showed that the flow follows a helical pattern in the meridional plane from the inlet to outlet port (Figure 3), which is induced by the centrifugal field of the impeller. This helical flow path cannot be directly described by the theories developed by Senoo [27] and Iversen [28].

The circulation-momentum exchange theory [29] states that the fluid gains momentum in the impeller and imparts this on to the slower moving fluid in the annular channel by circulation (Figure 2 ), increasing the pressure along the annular channel. The fluid then re-enters the impeller with a lower angular momentum. Despite its exclusion of the effects of drag and shear stresses, this theory has been recognised as sufficient to describe the inner flow mechanism and has formed the basis for most publications to date. A more detailed review of the investigation of the principle of operation of the RLR pump can be found in Karlsen \& Aggidis [30], however a summary is given in Table 1.

\subsection{Applications}

The primary method of use for the regenerative machine has been in the areas of liquid pumping [12, 21] and mainly for the delivery of water [27], however considerable modifications have occurred and applications have diversified. Today the RLR pump fulfils a whole range of duties [12] and the regenerative operating principle is also applied in the forms of compressors and turbines.

Regenerative machines are particularly useful for low-viscosity, clean liquid applications $[10,31]$ that require low flow rates and relatively high heads. Their inherent ability to provide multi-staging using only one impeller provides much higher heads compared to machines of similar tip speeds $[13,15]$. The head rise can be increased even further by incorporating several pumping stages on a common shaft [11]. 
The efficiency compares favourably with that of a centrifugal pump of very low specific speed but much less favourable for a large pump of high specific speed. They are an attractive option due to ease of manufacture, relatively low cost, compact design, stable operating conditions, excellent suction characteristics, high temperature capability without internal binding, reliability and excellent priming behaviour $[16,32]$, which are often more important than high efficiencies.

In general, the regenerative turbomachines find uses in the fields of biomedical pumping, condensate return, microturbines, automotive and aerospace fuel pumping, shipping and mining, sump service (clean water), laundry, car washers, breweries, spraying systems, chemical and food process industries, control, regulation of lubrication and filtering, booster systems, refineries and marine (potable water), refrigeration, cryogenic applications in space vehicles, hydrogen gas pipelines, accessory drives on aircraft and missiles, and other applications as regenerative blowers. In a low-pressure system a small booster pump, such as the RLR pump, can be used to obtain a high pressure flow and allow the remainder of the system to operate at a lower pressure and reduced power, resulting in reduced energy consumption [33]. RLR pumps are also used for petroleum pumping, viscous fluids, chemicals and caustic fluids, and they are particularly ideal for handling volatile or hot fluids due to the fact that they cannot become vapour locked $[7,10,15,29,32,34]$ and for this reason they found use in the V-2 rocket as shown in Figure 6.

\section{The German A-4 (V-2) rocket}
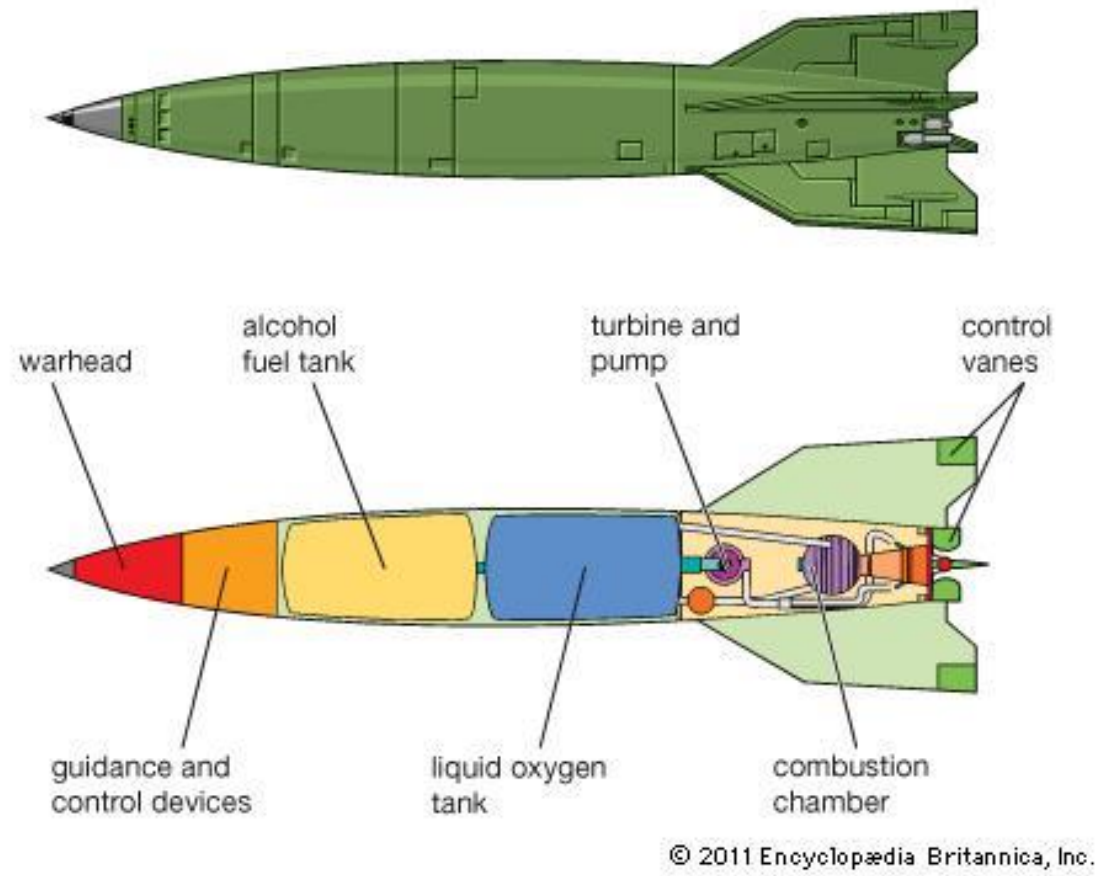

Figure 6: Schematic of a rocket using a regenerative fuel pump [35] 
Table 1: Comparison of methods used to analyse the regenerative operating principle (references refer to focused work within each mode of analysis)

\begin{tabular}{|c|c|c|c|c|c|c|c|}
\hline \multicolumn{3}{|c|}{ Operational Analysis } & \multirow[b]{2}{*}{ 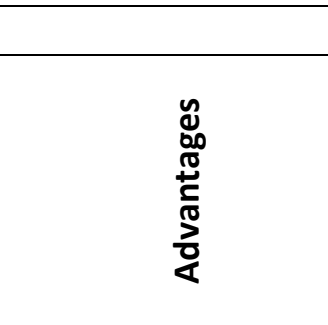 } & \multirow[b]{2}{*}{ 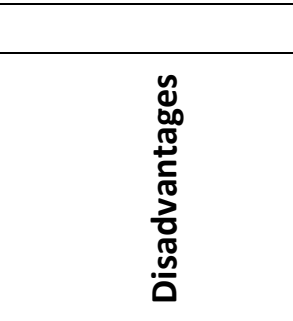 } & \multirow[b]{2}{*}{ 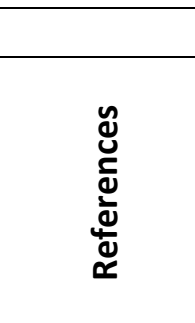 } & \multirow[b]{2}{*}{ 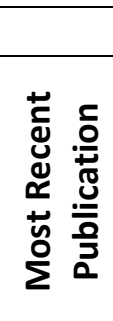 } & \multirow[b]{2}{*}{ Comments } \\
\hline $\begin{array}{ll}\frac{\omega}{0} & \frac{n}{n} \\
\frac{1}{0} & \frac{2}{N} \\
\frac{0}{2} & \frac{c}{4}\end{array}$ & 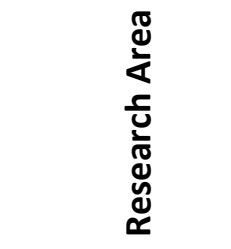 & 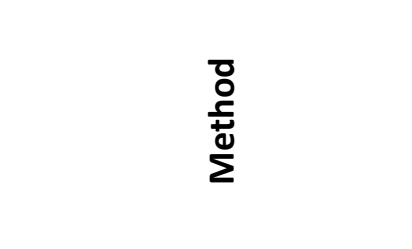 & & & & & \\
\hline \multirow{2}{*}{ 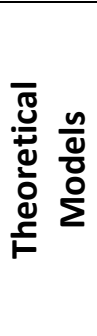 } & $\begin{array}{l}\text { Turbulent } \\
\text { Flow Theory }\end{array}$ & $\begin{array}{l}\text { Turbulent Mixing } \\
\text { Length } \\
\text { - Turbulent Shearing } \\
\text { Stress }\end{array}$ & \multirow[t]{2}{*}{ - Relatively fast } & \multirow{2}{*}{$\begin{array}{l}\text { - } \\
\text { - } \\
\text { Experimental } \\
\text { data } \\
\text { - Geometry } \\
\text { dependant }\end{array}$} & \multirow{2}{*}{$\begin{array}{l}{[6-8,16} \\
22,27-29 \\
32,36-42]\end{array}$} & \multirow[t]{2}{*}{2012} & \multirow{2}{*}{$\begin{array}{l}\text { Momentum exchange theory accepted as } \\
\text { most suitable } \\
\text { Recent modifications include entire flow } \\
\text { region and are independent of empirical } \\
\text { data } \\
\text { - Can be used for initial design generation }\end{array}$} \\
\hline & $\begin{array}{l}\text { Momentum } \\
\text { Exchange } \\
\text { Theory }\end{array}$ & $\begin{array}{ll} & \text { Circulation } \\
\text { - } & \text { Violent Mixing }\end{array}$ & & & & & \\
\hline 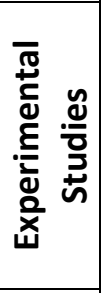 & $\begin{array}{l}\text { Flow } \\
\text { Visualisation }^{1}\end{array}$ & $\begin{array}{l}\text { - } \quad \text { Probe Measurement } \\
\text { - } \quad \text { Photographical } \\
\text { Recording } \\
\text { - } \quad \text { Laser \& Digital } \\
\text { Imaging }\end{array}$ & - Good visual aid & $\begin{array}{l}\text { - } \\
\text { consume } \\
\text { - Costly } \\
\text { - Inadequate } \\
\text { detail }\end{array}$ & $\begin{array}{c}{[12,22-} \\
25,43-44]\end{array}$ & 1995 & 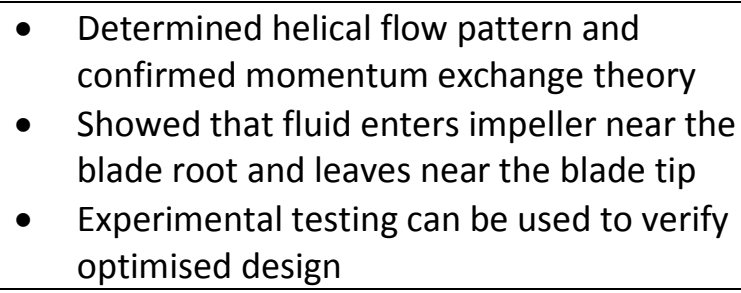 \\
\hline \multirow{2}{*}{ 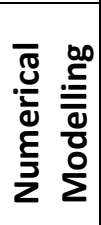 } & Steady State & - Frozen Rotor & \multirow{2}{*}{$\begin{array}{ll}\text { - } & \text { Less costly } \\
\text { - } & \text { High } \\
& \text { repeatability } \\
\text { - } & \text { Accurate }\end{array}$} & \multirow{2}{*}{$\begin{array}{l}\text { - } \text { Processing } \\
\text { limitations }\end{array}$} & \multirow{2}{*}{$\begin{array}{c}{[6,9,45-} \\
48]\end{array}$} & \multirow[b]{2}{*}{2014} & \multirow{2}{*}{$\begin{array}{l}\text { - Has taken the research to new levels } \\
\text { - Can be used for performance prediction } \\
\text { and parametric studies } \\
\text { - Ability to resolve local flow pattern }\end{array}$} \\
\hline & Transient & $\begin{array}{l}\text { Moving Reference } \\
\text { Frame }\end{array}$ & & & & & \\
\hline
\end{tabular}

\footnotetext{
1 References listed only include studies where experimental investigation has predominantly been used; they do not include experimental verification/comparison of theoretical/numerical modelling
} 


\section{Operational Advantages}

As illustrated in Figure 7 the RLR pump is well suited for high head-low capacity applications. High heads are concurrent with low flow rates. The circulatory velocity is higher at lower flow rates, resulting in this higher pressure rise. One effect of the subsequent high heads is a larger power requirement at these low flow rates, with the maximum required at shutoff. Therefore it can be demonstrated that the maximum efficiency of an RLR pump occurs at comparatively faster flow [15]. The efficiency curve features a sharp peak close to $50 \%$. The increased power demand at reduced flow rates can lead to overloading of electric drive motors designed to meet the demand at the duty point [49].

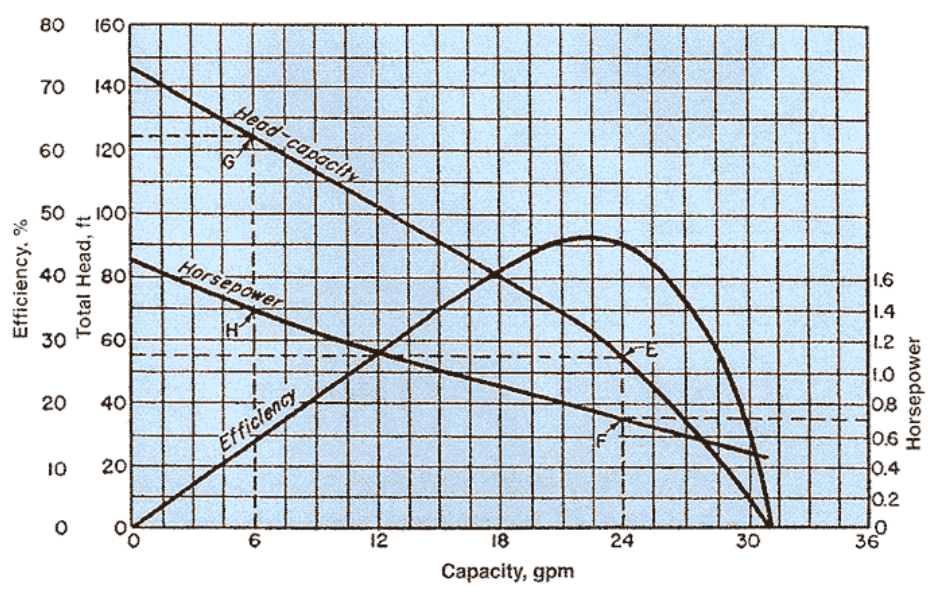

Figure 7: Performance curves for a typical regenerative pump [50]

\subsection{Low Specific Speed}

Despite the low efficiency, typically in the range of $35-50 \%[13,21]$, it is evident from Figure 8 that in the low specific speed range for delivery of small quantities of fluid at a high pressure, the RLR pump compares favourably with other more complex multistage rotodynamic machines. A comparative study [11] of a centrifugal and regenerative pump showed that the latter was capable of delivering a head nearly three times higher at a much greater efficiency, underlining the importance of pump selection. Based on data collected for the European Commission study in 2008 [1], better selection was estimated to give cost effective energy savings of $3 \%$ (or 1.1TWh pa) within a 15 year timeframe.

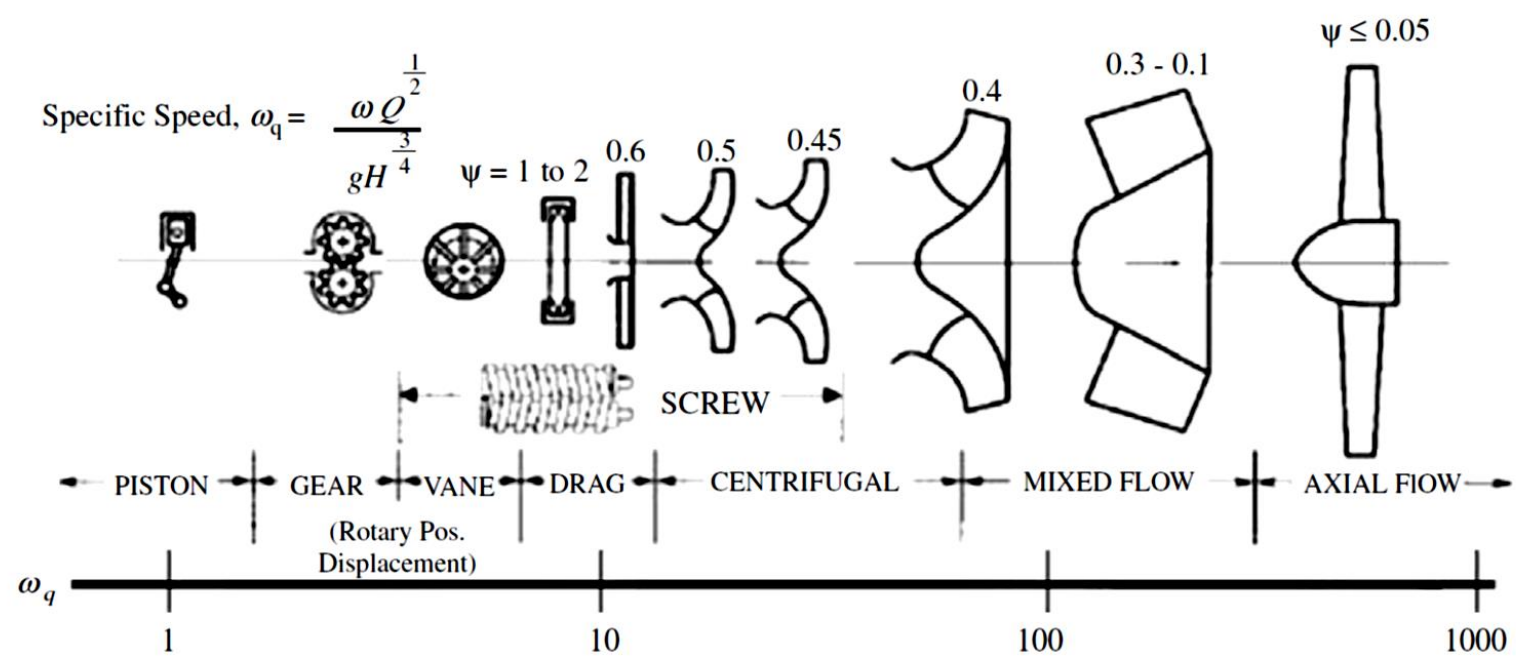

Figure 8: Specific speed classification for different types of pumps [6] 
It should however be noted that Hollenberg \& Potter [31] carried out an efficiency-specific speed study which indicated that higher efficiencies may be achievable at higher specific speeds.

\subsection{Self-Priming}

A typical horizontal centrifugal pump is sensitive to the presence of air and other gases in the pumped liquid, if the circumstances exceed the capability of the pump it might lose its prime or completely stop pumping $[5,51]$. RLR pumps on the other hand have excellent priming behaviour. The self-priming ability is ensured by correct arrangement of the inlet and outlet ports and external elbow joints, so that a portion of fluid always remains within the pump cavity. When the impeller begins to rotate, the remaining fluid is thrown around the casing to form a ring of liquid [5, 12], hence the alternative name liquid ring pump. Due to the gradual increase in pressure across the pump, any vapour bubbles occurring in the fluid will collapse gently over a relatively long time. This makes the RLR pump able to handle gases or a mixture of gas and liquid. Under the same conditions a centrifugal pump would be subjected to cavitation as the bubbles will collapse violently due to the sudden increase in pressure $[3,48]$. The amount of entrained gases the RLR pump can handle varies but numbers ranging from $20-50 \%$ have been reported $[48,51]$.

\subsection{Stable Operating Conditions}

As the gradient of the head-capacity curve is very steep, the RLR pump can maintain a relatively stable flow within a range of pressure fluctuations by throttling a valve. In contrast, typical centrifugal pumps have a parabolic shaped curve whereby the flow rate can change dramatically with pressure [51]. When a regenerative pump is applied as a gas compressor there is an additional advantage of no surge or instability [42].

\subsection{Low Cost}

The simplistic design featuring simple impeller blades and casing makes this type of pump easier and cheaper to manufacture than centrifugal pumps with intricate vanes and volutes. The RLR pumps are also more compact and require fewer stages than centrifugal pumps. Fewer components and less material indicate that the initial environmental impact, in form of raw material extraction and energy consumption during the manufacturing process, will in comparison be less for a RLR pump. This is an important factor to consider when assessing the life cycle cost in terms of energy usage.

\section{Performance Challenges and Next Steps}

The highest reported efficiency for any water-ring self-priming pump was measured by Crewdson in 1956 [21] at 50\%. The low efficiency can partly be assigned to the inherent fluid dynamic behaviour of the pump; the input power is used for developing the increase in head as well as being consumed by the circulatory flow through the impeller blades [15, 32, 52]. However, the pumping efficiency is significantly reduced when the circulatory flow decreases [21].

As much as $40-50 \%$ of the supplied power is believed to be consumed losses that are related to the geometry of the machine [53], of which losses due to slip make up the largest contribution [52, 54]. The performance of the RLR pump is also affected by other types of losses, such as shock losses, circulation losses, peripheral friction losses, losses at the inlet and outlet ports and leakage losses [5] $[12,15,53]$, which are explained in more detail in the subsequent sections.

Improvements in the design should be aimed at increasing the efficiency at high pressures and low flow rates due to the large corresponding increase in power consumption [55]. As soon as the flow rate increases, the fluid enters the impeller blades less frequently, with a lower degree of regeneration and a lower increase in pressure $[15,22,45]$. 


\subsection{Slip Losses}

Burton [12] described losses due to slip as "imperfect guidance of the flow by the blades". As the fluid traverses through the impeller the pressure between each adjacent blade will be different (due to the adverse peripheral pressure gradient) giving rise to a secondary circulation which forces the fluid exiting the blade to flow backward with respect to the rotational direction of the impeller $[5,8$, 16]. The slip loss increases with the peripheral pressure gradient $[15,56]$. The actual mean peripheral fluid velocity at the blade exit is less than the theoretical velocity based on the outlet blade angle (velocity triangle). It is this difference that provide the energy required to maintain the circulation [54]. The reduction in peripheral velocity is accounted for by introducing a slip factor which is defined as the ratio of the mean peripheral velocity to the velocity of the blading $[8,54]$. To reduce these losses, Sixsmith [54] suggested to increase the inlet blade angle and reduce the outlet angle. The amount of slip at zero head gives an indication of the drag (or 'hold') which the blades have on the fluid [21].

\subsection{Shock Losses}

In contrast to the slip effect, shock (or incidence) losses occur at the entry to the blades and predominantly at operating conditions outside the duty point. It is believed that the loss is due to the difference in angle between the blade and the flow when the fluid enters the blade $[5,16,42]$ as a result of the difference in angular momentum between the slower moving fluid in the channel and the faster moving fluid in the impeller. The shock effect is accounted for by an incidence factor which is defined as the ratio of the mean peripheral fluid velocity at the blade inlet to the velocity of the blading [8].

\subsection{Circulation Losses}

During operation the RLR pump experiences hydraulic losses as the flow circulates between the impeller blades and the annular channel $[5,15,53]$. This type of loss can be separated into three loss mechanisms [7-8, 16, 42]. The first occurs due to turning of the fluid in both the annular channel and in the blades. More specifically the channel turning loss can be assigned to the sharp corners of the channel. The second loss mechanism is due to the mixing of faster moving flow in the impeller with the slower moving flow in the annular channel. Thirdly, when the flow circulates through the impeller it will experience a sudden change in flow area when it enters or leaves the blades.

\subsection{Peripheral Friction Losses}

Peripheral head losses are caused by the natural curvature of the annular channel which gives rise to wall shear stresses $[16,39]$. It can be evaluated in the same way as classical pipe losses occurring in bends, contraction, expansion and straight duct [7-8, 38].

\subsection{Inlet and Outlet Losses}

The losses that occur at the inlet and outlet ports of the pump are primarily caused by the difference in the flow area between the ports and the channel [7], which causes rapid changes in fluid velocities.

\subsection{Leakage Losses}

There are two major types of leakage losses occurring in the RLR pump: firstly through the axial and radial clearances between the impeller and the pump casing and secondly between the inlet and outlet ports across the stripper. The latter is the largest and has a greater effect on the pump 
performance $[5,12,28,32,41,54]$. During pumping operation, the high-pressure fluid near the discharge port, assisted by the impeller blades which are moving in the same direction, will attempt to leak back through the stripper clearance to the low-pressure suction port. This occurs due to the pressure difference existing between the fluid in the impeller blades and the fluid in the side channel and due to the drag of the impeller. To avoid excessive backflow the stripper clearance must be kept to a minimum. However, Sixsmith \& Altmann [52] believed that only small improvements in performance could be gained. Sixsmith [54] later identified that mulitstaging could reduce the loss of efficiency due to the backflow by nearly a half. Burton [12] also identified a third type of leakage loss, that occurring between the side channel and the central blade "pocket" (area between two successive blades), however it turned out to only have a minor effect on the pump performance compared with the losses through the stripper section.

\subsection{Operational Wear}

RLR pumps are generally very robust compared to other pumps and offer long service life, however performance challenges can arise due to operational wear of internal components. Although it is important to ensure tight clearances for optimum head and efficiency [31], it does not come without its price. Abrasives in the pumped fluid will cause rapid wear on the impeller and casing, making the RLR pumps poor at handling solids of any appreciable size [10,31]. This kind of fluid contamination has a tendency to cause premature failure in mechanical seals and erosion of the impeller blades, as particles get stuck between the impeller and the casing [57]. For different applications it becomes important to identify parameters that affect component wear and consequently performance as these will directly affect how the energy consumption will change over time, with the added benefit of improving pump reliability. Ensuring that proper maintenance of existing pumps is carried out can also make substantial energy savings. Replacing worn pump parts will reduce internal leakage and maintain pump efficiency. An added benefit of this is the reduction of the life cycle impact on the environment by refurbishing rather than replacing the whole pump.

Due to the gradual increase in pressure from the suction to the discharge side, there is a radial load on the impeller $[5,21]$. It is of key importance that the pump reaches a pressure equilibrium within a reasonable period of time to ensure proper axial balancing of the impeller [57]. Until this point the impeller is sensitive to sudden changes in pressure, due to pressure valves or other conditions, which could cause it to rub against the casing. During operation some deformation of the impeller could take place due to increase in temperature, adversely affecting the clearances and alignments within the pump. In comparison, the radial clearance is typically less subject to wear [32].

\section{Geometry and Design Optimisation}

The design of the regenerative machine has evolved over many decades. Similar to the concept of the paddlewheel [18] most of the earlier designs of regenerative turbomachines consisted of a basic geometrical configuration with simple vanes either cast or machined into the impeller. Throughout the years researchers have investigated the correlation between performance and geometrical features, focusing on the design of the blade shape, the side channel, the inlet and the outlet.

\subsection{Blade Configurations}

The design of the impeller blades has a significant effect on the performance due to the influence on the fluid flow and the energy transfer to the fluid [55], this is perhaps why most of the design improvements have centred on the geometry of the blading. The impeller blades of regenerative turbomachinery can have different profiles including radial, non-radial, semi-circular and aerofoil blades. These profiles can be configured into a single- or double-sided impeller. The latter, also sometimes referred to as a webbed impeller as it features a "web" between the blades, promotes equal pressure distribution on both sides, which in turn makes the impeller self-adjust axially and reduces the chances of wear [5]. This is probably why this type is the most common form used for 
commercial units $[28,58]$. Symmetrical blade profiles have also been shown to increase the efficiency due to improved energy transfer between the impeller and the fluid [52]. This can be assigned to the reduction of so called "dead zones" of fluid between adjacent blades which do not take part in the circulation [59].

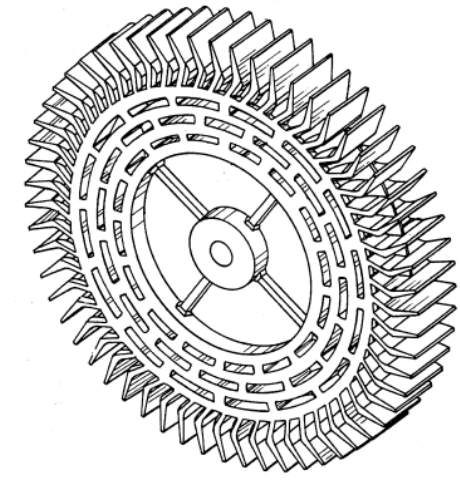

(a)

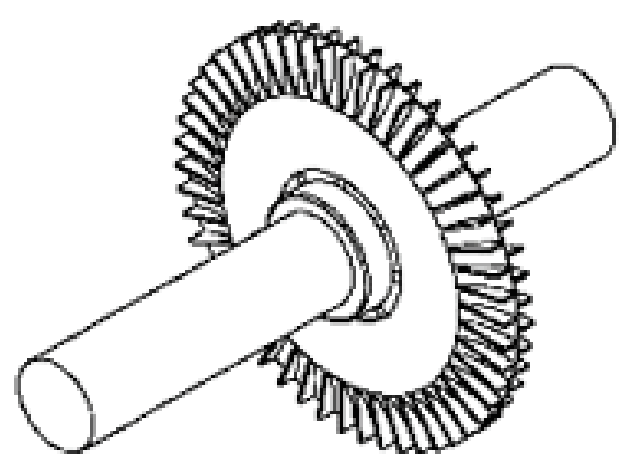

(b)

Figure 9: (a) Single-sided [60], (b) double-sided regenerative pump impeller [61]

The most widely used blade profile is the straight radial blade (Figure 10a), which is perpendicular to the perimeter of the impeller and can rotate in either direction [27-29, 37, 55, 62]. Different variations of this profile exist. Particularly, a circular shape in the meridional section (applying a radii to the corners) has been found to reduce turbulence and promote better flow circulation by reducing the incidence angle [5, 63-64].

Non-radial blades have a similar profile but can either be forwardly or backwardly inclined in relation to the impeller's direction of rotation $[12,44,62,65-69]$. A recent experimental study found that the head performances of both forward and backward bladed impellers deteriorate as the blade angle increases, underlining that straight radial blades are more efficient in comparison [62]. However, out of the two, the forwardly inclined blade performs better [65].

The chevron shaped blades (Figure 10b), angled with respect to the axial direction, are in some ways similar to a Pelton turbine as the fluid is scooped up by the blade shape, rendering them more efficient if rotated in the direction of the blade inclination [30]. Chevron impellers have not been subjected to as much study as radial blades, but have been found to provide improved performance compared with radial blades. This is due to the reduction in incident loss at the blade inlet and subsequently higher circulation [12, 21, 32, 43-44]. Furthermore, the radial clearance is reduced as the chevron impeller features a continuous surface around the periphery circle, which limits the leakage losses [70]. Both Burton [70] and Badami [32] found that the head produced by the chevron bladed impeller is around twice that of a radial blade impeller. One study found the optimum chevron angle of around $30^{\circ}$ [62]. While another study [16] on the other hand determined it to be between $45^{\circ}$ and $60^{\circ}$, which makes it rather difficult to from a design point of view to select an ideal angle. As is the case for centrifugal pumps, the parametric design of the RLR pump is dependent upon the operational parameters.

Only limited research exists into semi-circular profile blades (Figure 10c) [31, 65], they have however been shown to have a complete absence of the dead zones and a minimum wetted surface perimeter in the case of viscous liquids [59].

Aerofoil blades (Figure 10d) have a circular contour and project axially into the channel, they have mostly been used in regenerative compressor designs [52, 54, 71-72]. Aerofoil profiles are usually fitted with a core for guiding the flow and reducing the vortex formation at the blade tips [52]. Sixsmith and Altmann [52] found that the aerofoil blades produced more pressure rise than any other blade shapes. Although the efficiency was improved compared to the radial blade impeller, the 
efficiency remained around $40 \%$. They suggested to use properly contoured aerofoil blading to increase the efficiency further. As with other blade profiles, symmetrical blading gave a higher efficiency.

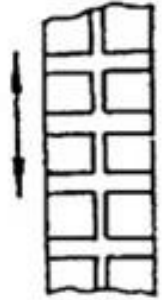

(a)

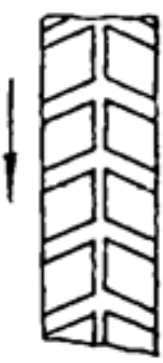

(b)

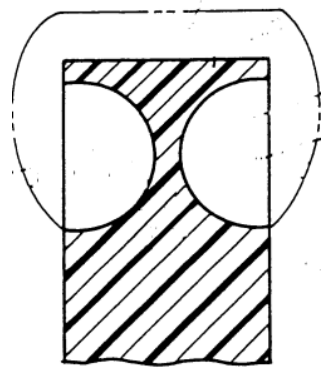

(c)

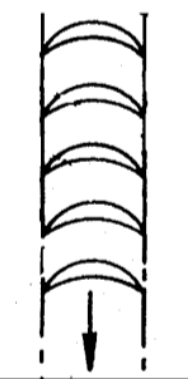

(d)

Figure 10: (a) Straight radial blades, (b) symmetrical chevron blades, (c) meridional geometry of semi-circular blades, (d) meridional section of aerofoil blades [73-74]

Interestingly, Shirinov and Oberbeck [75] found that aerofoil blades are superior to the other blades types tested for pressures higher than $20 \mathrm{kPa}$, while symmetrical chevron blades outperform in the $100 \mathrm{~Pa}$ to $20 \mathrm{kPa}$ range because they allow the best momentum transfer from the impeller to the fluid in the side channel.

Meakhail \& Park [42] carried out a CFD study to investigate the complex flow field of a regenerative pump using an impeller with twisted blades, which combines a chevron inlet blade angle and a backwardly inclined angle at the side-blade exit. They were able to show that part of the fluid leaves the impeller at the side of the blade, in contrast to the assumptions made by Wilson et al. [29]. Based on their results they determined that the side-blade exit angle has a significant effect on the performance. Angles less than $90^{\circ}$ gave higher efficiencies due to the fact that the flow angle is close to the blade angle, which reduces or eliminates vortex formation behind the blade.

Regardless of blade profile, blade angles matching the fluid angles provides a better performance as the fluid will have a smoother entry and exit of the blades [54].

\subsection{Number of Blades}

Some researchers $[21,28,37,46,55,65,76]$ have also successfully improved the impeller performance by varying the number of impeller blades. The number of blades has an effect on the head developed by the pump [32] due to the effect on the slip factor [5, 28, 77]. As the number of blades is reduced the losses due to slip are increased by a large amount. Raheel \& Engeda [16] did a study involving radial blade profiles and concluded that 75 to 90 blades should mitigate this problem. However, a further increase could have an adverse effect on the circulation between the blades as well as increasing the friction losses.

However, as with all design criteria, the exact number of blades will vary for different designs and conditions. Iversen [28] and later Shirinov \& Oberbeck [75] determined that the optimal size, number and spacing of the blades are strongly related to the diameter of the impeller and the size of the side channel. For an increase in the cross-sectional area of the side channel, the optimum impeller will feature larger but fewer blades in a more sparse formation. On the other hand, if the diameter of the impeller is increased while the side channel area is unchanged, the ideal number of blades will increase relative to the diameter.

\subsection{Impeller Diameter}

Losses arising from shock and slip are mainly due to misalignment of the blade and fluid angles. Therefore it comes as no surprise that these losses are also affected by the impeller tip radius. 
Raheel \& Engeda [16] found that these could be minimised by increasing the impeller tip radius and decreasing the hub radius.

\subsection{Axial and Radial Clearance}

Research has shown that a decrease in axial clearances will provide an increase in efficiency, due to reduction in backflow from the high-pressure discharge to the low-pressure suction. There is no definite number for the optimum clearance. Sixsmith [54] determined it to less than $0.3 \mathrm{~mm}$ to prevent excessive backflow. Through comparison of experimental and theoretical results of different axial clearance values $(0.01-0.08 \mathrm{~mm}$ ) Badami [32] found that $0.01 \mathrm{~mm}$ provided a higher head and better efficiency.

Less attention has been given to the radial clearance. Raheel \& Engeda [16] found that the radial clearance has an effect on losses due to shock, turning of the blades, and channel and blade mixing. These were minimised by increasing the radial clearance.

\subsection{Side Channel}

The shape and cross-sectional area of the side channel have an effect on the performance of the pump. An unnecessary amount of power is lost in sustaining the circulatory flow against the resistance due to fluid friction and turbulence (caused by violent mixing of slower and faster moving fluids) in the channel [52]. It becomes important to reduce this resistance to a minimum. Particularly, a circular cross-section or rounded shape assists with the circulatory flow and reduces turbulence $[5$, 54]. Studies [32, 37] have also shown that an increase in cross-sectional area the inlet region initiates the circulatory flow and improves head development and efficiency. In addition, increasing the width of the open area of the side channel decelerates the circulatory flow and reduces the kinetic energy, which in turn provides the power required to support the circulation against the friction [52]. The depth of the side channel also has an effect on shock and channel turning losses, notably a sharp drop in efficiency occurs for large channel depths [16].

Attempts have also been made to control the circulatory flow by including a central core in the side channel $[21-22,78]$. Crewdson [21] soldered a thin brass strip along the middle of the side channel, dividing it into two parts, to affect any radially inward flowing circulatory flow. From the performance curves established, he was able to conclude that the pumping efficiency was significantly reduced with decreasing circulatory flow.

Based on previous research carried out by Pfaff [78], Burton [70] investigated the use of fixed return blades at the root of the side channel and near the tip of the impeller, both improved the performance by altering the torque balance between the impeller and the side channel and by guiding the fluid back into the impeller blade area, respectively.

\subsection{Stripper}

The stripper is situated between the suction and discharge ports and plays an important role as it forces the fluid to leave through the outlet rather than flowing back to the inlet region. Limited research exists on the stripper region and investigation into the effects of key parameters such as its length and form should be carried out. A small stripper angle (the angle between the inlet and the outlet ports) gives a corresponding larger pumping area and increases the interaction time between the fluid and the impeller. However, more flow will leak through the stripper gap from the discharge to the suction [13].

\subsection{Suction \& Discharge Ports}

The design of the inlet controls how the fluid will interact with the impeller blades. The majority of RLR pumps feature straight, vertical ports leading into the channel in a radial direction [6], however studies have shown that this arrangement induces large losses at the inflow [9]. This can be mitigated by realigning the fluid flow so it enters and leaves the pump in an axial direction. This will 
ensure that the fluid is directed towards the inlet edge of the impeller blades with the velocity required to ensure smooth entry at the design pressure [54, 79]. Senoo [36] found that the pump performance is sensitive to the pressure drop that occurs when the fluid enters through the inlet port, indicating that the shape and position of the inlet are parameters that should be investigated further. Sixsmith also [54] suggested having a tapered diffuser after the discharge port to reduce the velocity of the fluid and translating some of the kinetic energy to an extra increase in pressure. The axial arrangement of the suction and discharge ports proposed by Quail et al [6] will also minimise axial thrust on the impeller. 
Table 2: Summary of performance challenges and geometrical design optimisation

\begin{tabular}{|c|c|c|c|c|c|}
\hline \multicolumn{3}{|c|}{ Geometry and Performance } & \multirow[b]{2}{*}{ 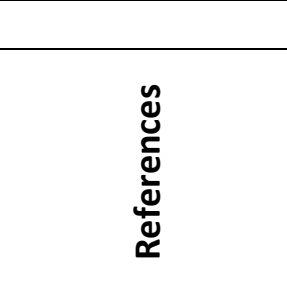 } & \multirow[b]{2}{*}{ 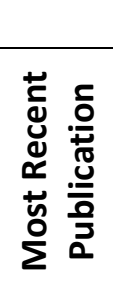 } & \multirow[b]{2}{*}{ Comments } \\
\hline Geometry & Performance Effects & $\begin{array}{l}\text { Geometrical } \\
\text { Parameter / } \\
\text { Configuration }\end{array}$ & & & \\
\hline Impeller & $\begin{array}{l}\text { - } \quad \text { Axial thrust } \\
\text { - } \text { Misalignment of } \\
\text { blade and fluid angles }\end{array}$ & $\begin{array}{l}\text { - } \quad \text { Single-sided } \\
\text { - } \quad \text { Double-sided } \\
\text { - } \quad \text { Diameter }\end{array}$ & {$[5,16,28,58]$} & 2005 & $\begin{array}{l}\text { Double-sided promotes equal pressure distribution, } \\
\text { reduces chances of wear } \\
\text { - Increasing impeller tip radius and decreasing hub } \\
\text { radius mitigate shock and slip losses }\end{array}$ \\
\hline Blade & $\begin{array}{l}\text { - Misalignment of } \\
\text { blade and fluid angles }\end{array}$ & $\begin{array}{ll}\text { - } & \text { Profile } \\
\text { - } & \text { Angle } \\
\text { - } & \text { Number }\end{array}$ & $\begin{array}{c}{[12,16,21,27-} \\
29,31,32,37 \\
42,44,46,52 \\
54-55,58-59,62- \\
70,75,77]\end{array}$ & 2014 & $\begin{array}{l}\text { - Symmetrical profiles more efficient } \\
\text { - } \text { Aerofoil and chevron blades perform better } \\
\text { - Blades matching fluid angles ensure smooth entry and } \\
\text { exit of fluid }\end{array}$ \\
\hline Clearance & $\begin{array}{ll}\text { - } & \text { Backflow } \\
\text { - } & \text { Entry to blade } \\
\text { - } & \text { Circulation } \\
\end{array}$ & $\begin{array}{ll}- & \text { Axial } \\
\text { - } & \text { Radial }\end{array}$ & {$[16,32,54,80]$} & 2009 & $\begin{array}{l}\text { - } \quad \text { No definite number for optimal clearance } \\
\text { - } \quad \text { Less attention given to radial clearance }\end{array}$ \\
\hline Side-channel & $\begin{array}{ll}\text { - } & \text { Resistance } \\
\text { - } & \text { Turbulence } \\
\text { - } & \text { Circulation }\end{array}$ & $\begin{array}{ll}\text { - } & \text { Shape } \\
\text { - } & \text { Width } \\
\text { - } & \text { Depth } \\
\end{array}$ & $\begin{array}{c}{[5,16,21-22,32} \\
37,54,70,78]\end{array}$ & 2005 & $\begin{array}{l}\text { - Rounded shape assist circulation and reduces } \\
\text { turbulence } \\
\text { - } \quad \text { Increasing width reduces power consumption loss } \\
\text { - } \quad \text { Return vanes for improved fluid guidance }\end{array}$ \\
\hline Stripper & - Backflow & $\begin{array}{l}\text { - } \text { Length } \\
\text { - } \text { Width } \\
\text { - } \quad \text { Shape }\end{array}$ & [13] & 2010 & - Limited research exists \\
\hline $\begin{array}{l}\text { Suction / } \\
\text { Discharge }\end{array}$ & $\begin{array}{l}\text { - Initial fluid interaction } \\
\text { - } \quad \text { Axial thrust }\end{array}$ & $\begin{array}{ll}\text { - } & \text { Alignment } \\
\text { - } & \text { Shape } \\
\text { - } & \text { Auxiliary fittings }\end{array}$ & {$[6,9,36,54,79]$} & 2012 & $\begin{array}{l}\text { - Axial arrangement ensures alignment of fluid } \\
\text { - Diffuser at discharge to reduce velocity }\end{array}$ \\
\hline
\end{tabular}




\section{Conclusions}

This paper offers an extensive review into the development, performance challenges and design improvements of RLR pumps in order to provide some useful insight on future research and next steps.

Despite the fact that the exact principle of operation of the RLR pump has been a phenomenon not fully understood, it has nevertheless been widely applied for over a century in areas of liquid pumping. Its interesting design makes it suitable for a wide range of applications, particularly when efficiency is of less importance than stable operating conditions. Due to the low specific speed, it is an excellent choice when small quantities of liquid are to be delivered against high heads. It is also ideal for pumping volatile or hot fluids as it cannot become vapour locked. The fairly low efficiency does bring into question the benefits and drawbacks of different types of pumps in a climate of reducing the global energy consumption. Appropriate selection of pumps can greatly reduce $\mathrm{CO} 2$ emissions and incur energy savings. The RLR pump has in some cases been proven to be a much more efficient alternative to the traditional centrifugal pump.

The main challenges identified for further improvement in the performance of RLR pumps include the ability to fully comprehend the highly complex and turbulent internal flow field and the associated losses that occur. Early research made an attempt at this through various mathematical models, however they could not fully represent the three-dimensional helical flow pattern and also required experimental correction data. Flow visualisation studies proved more successful in illustrating the nature of the flow. In parallel to this work, the design of the regenerative machine has evolved over many decades and improvements have been made in the design, particularly in the shape and number of impeller blades. Despite these efforts the efficiency of RLR pumps still remain low, however it is possible that maximum efficiency might be physically limited to efficiencies in the region of $50 \%$ and that future developments should therefore focus on improving other aspects of the pump, such as reliability and self-priming.

Another aspect that requires more investigation is the interaction of the different loss reduction methods to determine whether mitigating one type of loss, through optimisation of design or operation, will have a negative effect on another. It might be that the optimal RLR pump will vary greatly with different requirements.

Today we have the tools available to obtain a comprehensive representation of the flow field in computational modelling techniques, which grants the ability to make incremental changes in an iterative fashion. Models can be continually refined and then verified with experimental testing in a shorter time frame. Research and development of regenerative turbomachinery has grown in volume and speed due to the availability of these techniques in recent times.

The conclusions drawn from the findings in this paper form the basis of novel research into parameters that affect the performance of a newly built RLR pump as well as achieving a better understanding of its operational life cycle. A holistic approach to improving the efficiency must be taken. Pump performance can be greatly affected by wear and improved reliability will ultimately reduce the overall energy consumption from "cradle to grave".

\section{Acknowledgements}

The authors would like to thank Lancaster University Renewable Energy and Fluid Machinery Group and Gilbert Gilkes and Gordon Ltd. 


\section{References}

[1] European Commission, "Study on improving the energy efficiency of pumps," February 2001, AEAT-6559/ v 5.1

[2] H. Vogelesang, "An introduction to energy consumption in pumps," World Pumps, pp. 28-31, January 2008.

[3] S. Mueller, "Consider regenerative pumps for low-flow/low-NPSH applications," Hydrocarbon Processing, pp. 55-7, August 2004.

[4] R. Mulley, Flow of Industrial Fluids - Theory and Equations, USA: CRC Press, 2004.

[5] S. Lazarkiewicz and A. Troskolanski, "Self-Priming Pumps," in Impeller Pumps, Warsawa, Pergamon Press Ltd., 1965, pp. 567-90.

[6] F. Quail, T. Scanlon and A. Baumgartner, "Design study of a regenerative pump using onedimensional and three-dimensional numerial techniques," Eur J of Mech B/Fluids, vol. 31, pp. 181-7, 2012.

[7] F. Quail, T. Scanlon and M. Stickland, "A One-Dimensional Numerical Model for the Momentum Exchange in Regenerative Pumps," J Eng Gas Turbines Power, vol. 133, no. 9, 2011.

[8] I. Yoo, M. Park and M. Chung, "Improved momentum exchange theory for incompressible regenerative turbomachines," Proc. IMechE, Part A: J Power and Energy, vol. 219, no. 7, pp. 56781, 2005.

[9] F. Quail, T. Scanlon and M. Stickland, "Design optimisation of a regenerative pump using numerical and experimental techniques," Int J Number Method H, vol. 21, no. 1, pp. 95-111, 2011.

[10] H. Biheller, "Special Variants of Dynamic Pumps," in Pump Handbook, I. Karassik, W. Krutzsch, W. Fraser and J. Messina, Eds., USA, McGraw-Hill, 1976, pp. 2.202-3.

[11] F. Quail, M. Stickland and A. Baumgartner, "Design study of a novel regenerative pump using experimental and numerical techniques," in 11th European Fluid Machinery Congress 12-15 September 2010, Edinburgh, 2010.

[12] J. Burton, "A Theoretical and Experimental Analysis of the Flow in Regenerative Pumps and Turbines," Ph.D. Thesis, University of Southampton, UK, 1966.

[13] F. Quail, T. Scanlon and M. Strickland, "Development of a regenerative pump impeller using rapid manufacturing techniques," Rapid Prototyping J, vol. 16, no. 5, pp. 337-44, 2010.

[14] P. Cooper, "Sealless integral-motor pump with regenerative impeller disk". USA Patent 628015728, 2001.

[15] M. Raheel and A. Engeda, "Current Status, Design and Performance Trends for the Regenerative Flow Compressors and Pumps," in ASME Paper No IMECE2002-39594, 2002.

[16] M. Raheel and A. Engeda, "Systematic Design Approach for Radial Blade Regenerative Turbomachines," J Propul Power, vol. 21, no. 5, pp. 884-92, 2005.

[17] MTH Pumps, "Regenerative Turbine Principles," 2014. [Online]. Available: http://www.mthpumps.com/turbine.html. [Accessed 14 March 2014].

[18] J. Imray, "The Helical Pump," in Proc. IMechE, Cardiff, 1874, vol. 25, no. 1, pp. 281-96.

[19] E. Molloy, Ed., Modern pumps: A comprehensive survey of modern pumping equipment and practice, Newnes, 1953, p. $70 f$.

[20] H. Addison, A Treatise on Applied Hydraulics, 4th ed., London: Chapman and Hall, 1954.

[21] E. Crewdson, "Water-ring self-priming pumps," Proc. IMechE, vol. 170, no. 1, pp. 407-25, 1956.

[22] H. Engels, "Investigations of Ring pumps," Tech Hoch Hannover, Germany, 1940.

[23] J. Bartels, "Performance of a peripheral pump," Polytechnic Institute of Brooklyn, New York, USA, 1947. 
[24] G. Lutz, "Experimental investigation of the pressure distribution in a regenerative turbine pump," Senior Thesis, Massachusetts Institute of Technology, USA, 1953.

[25] L. Lazo and T. Hopkins, "Theoretical and experimental analysis of a regenerative turbine pump," Senior Thesis, Massachusetts Institute of Technology, USA, 1953.

[26] G. Aggidis, "Regenerative liquid ring pumps experimental modelling and flow visualisation," Internal Research Report, Gilbert Gilkes \& Gordon Ltd, Kendal, UK, 2002.

[27] Y. Senoo, "Theoretical research on friction pump," Institute of Fluid Engineering, vol. 5, no. 1, pp. 23-48, 1948.

[28] H. Iversen, "Performance of the periphery pump," Trans ASME, vol. 77, pp. 19-28, 1955.

[29] W. Wilson, M. Santalo and J. Oelrich, "A theory of the fluid dynamic mechanism of regenerative pumps," Trans ASME, vol. 77, no. 8, pp. 1303-16, 1955.

[30] N. Karlsen and G. Aggidis, "Review and state of the art of regenerate liquid ring pumps," in 12th European Fluid Machinery Congress 6-7 October 2014, Edinburgh, UK, 2014.

[31] J. Hollenberg and J. Potter, "An Investigation of Regenerative Blowers and Pumps," ASME J. Eng. Ind. Trans, vol. 101, no. 2, pp. 147-52, 1979.

[32] M. Badami, "Theoretical and experimental analysis of traditional and new periphery pumps," SAE Technical Paper No 971074, 1997.

[33] J.C. Cone, "Pump energy conservation techniques", in Proc. $9^{\text {th }}$ Turbomachinery Symposium, Texas A \& M University, pg. 83-101, 1980.

[34] MEPCO, "Regenerative Turbines," 2014. [Online]. Available: http://www.mepcollc.com/products.html. [Accessed 5 Jul 2015].

[35] Encyclopaedia Britannica, "Regenerative Pump," Encyclopaedia Britannica, Inc, 2011. [Online]. Available: http://www.britannica.com/technology/regenerative-pump. [Accessed 201512 10].

[36] Y. Senoo, "A comparison of regenerative pump theories supported by new performance data," Trans ASME, vol. 78, pp. 1091-1102, 1956.

[37] Y. Senoo, "Influences of the suction nozzle on characteristics of peripheral pump and an effective method of their removal," Rep Res Inst Appl Mech, vol. 3, no. 11, 1954.

[38] J. Song, A. Engeda and M. Chung, "A modified theory for the flow mechanism in a regenerative flow pump", Proc. IMechE, Part A: J Power Energy, vol. 217, no. 3, pp. 311-, 2003.

[39] D. Andrew, "The calculation of flow in regenerative turbomachines by a streamline curvature method," Proc. IMechE, Part A: J Power Energy, vol. 204, no. 2, pp. 121-9, 1990.

[40] J. Oeirich, "Development of an analysis of a regenerative pump," Senior Thesis, Massachusetts Institute of Technology, USA, 1953.

[41] D. Burton, "Review of regenerative compressor theory," in Rotat. Machin. Gas-Cooled Reactor Applic, Tennessee, 1962.

[42] T. Meakhail and S. Park, "An improved theory for regenerative pump performance," Proc. IMechE, Part A: J Power Energy, vol. 219, no. 3, pp. 213-22, 2005.

[43] M. Hübel, B. Blättel and W. Strohl, "Investigation on Fluid Mechanics of the Regenerative Pump Used in Gasoline Injection Systems," SAE Technical Paper 950077, pp. 131-9, 1995.

[44] J. Burton, "The prediction and improvement of regenerative turbo-machine performance," in BHRA Members 9th Annual Members Conference September 1967, Paper SP 915, Cranfield, 1967.

[45] M. Badami and M. Mura, "Comparison between 3D and 1D simulations of a regenerative blower for fuel cell application," Energy Convers Manage, vol. 55, pp. 93-100, 2012.

[46] V. Karenth and N. Sharma, "CFD Analysis of a Regenerative Pump for Performance Enhancement," in World Conference on Applied Sciences, Engineering \& Technology 27-29 September 2014, Kathmandu, Nepal, 2014. 
[47] V. Weise and J. Beilke, "3D flow in a peripheral fan," International Journal of Computer Applications in Technology, vol. 11, no. 3-5, pp. 203-210, 1998.

[48] F. Quail, M. Stickland and T. Scanlon, "Numerical and experimental design study of a regenerative pump," In: Current Themes in Engineering Science. AIP, pp. 165-80, London, 2010.

[49] B. Nesbitt, Handbook of Pumps and Pumping: Pumping Manual International, Oxford: Elsevier and Rolls \& Associates, 2006, p. 29.

[50] Roth Pump Company, "Bulletin 1C99: Regenerative Turbine Chemical Pumps for the Process Industry," Roth Pump Company, $2015 . \quad$ [Online]. Available: http://www.rothpump.com/technical.html. [Accessed 6 August 2015].

[51] T. Marty, "Side Channel Sealless Magentic Drive Pumps," Pumps \& Systems, pp. 2-4, November 2012.

[52] H. Sixsmith and H. Altmann, "A regenerative compressor," Trans ASME, vol. 99, no. 3, pp. 63747, 1977.

[53] A. Engeda, "Flow analysis and design suggestions for regenerative flow pumps (Keynote)," in ASME/JSME 4th Joint Fluids Summer Engineering Conference 6-10 July 2003, Hawaii, USA, 2003.

[54] H. Sixsmith, "The theory and design of a regenerative compressor," Wallington, Surrey, 1981.

[55] A. Shirinov and S. Oberbeck, "Optimisation of the high vacuum side channel pump," in 7th International Conference on Compressors and their Systems 5-6 September 2011, London, UK, 2011.

[56] A. El Hag, "A theoretical analysis of the flow in regenerative pumps," University of Bath, UK, 1979.

[57] Lytron, "Maintaining your Regenerative Turbine Pump," 2015. [Online]. Available: http://www.lytron.com/Tools-and-Technical-Reference/Application-Notes/RegenerativeTurbine-Pumps. [Accessed 14 Aug 2015].

[58] M. Shimosaka, "Research on the characteristics of regenerative pump," Bull of JSME, vol. 3, no. 10, pp. 191-9, 1960.

[59] I. Zozulya, "Effect of the indentation profile shape on the regenerative-pump parameters," Chemical and Petroleum Engineering, vol. 18, no. 6, pp. 224-6, 1982.

[60] N. Moss and R. Czarnowski, "Extended range regenerative pump with modified impeller and/or housing". USA Patent U.S. Patent 5,527,149, 18 Jun 1996.

[61] Lytron, "Regenerative Turbine Pumps," Lytron, 2015. [Online]. Available: http://www.lytron.com/Tools-and-Technical-Reference/Application-Notes/RegenerativeTurbine-Pumps. [Accessed 7 Aug 2015].

[62] W. Choi, I. Yoo, M. Park and M. Chung, "Experimental study on the effect of blade angle on regenerative pump performance," Proc. IMechE, Part A: J Power Energy, vol. 227, no. 5, pp. 58592, 2013

[63] D. Dewitt, "Rational design and development of the regenerative pump," Senior Thesis, Massachusetts Institute of Technology, 1957.

[64] S. Kang, H. Lim and S. Ryu, "Performance evaluation of a regenerative pump of small size," in ASME Heat Transfer/Fluids Engineering Summer Conference 11-15 July 2004, Charlotte, North Carolina, USA, 2004.

[65] G. Grabow, "Influence of the number of vanes and vane angle on the suction behaviour of regenerative pumps. In: , Budapest, Hungary, 14-20 September 1975: pp. 351-64.," in Proceedings of 5th Conference on Fluid Machinery 14-20 September 1975, Budapest, Hungary, 1975.

[66] S. Yamazaki, Y. Tomita and T. Sasahara, "Research on the performance of the regenerative pump with non-radial vanes," Bull of JSME, vol. 15, no. 81, pp. 337-43, 1972.

[67] S. Yamazaki and Y. Tomita, "Research on the performance of the regenerative pump with non 
radial vanes," Bull of JSME, vol. 14, no. 77, pp. 1178-86, 1971.

[68] S. Yamazaki and T. Y, "Research on the performance of the regenerative pump with non-radial vanes," Bull of JSME, vol. 17, no. 104, pp. 459-66, 1974.

[69] Y. Tomita, S. Yamazaki and T. Sasahara, "The scale effect and design method of the regenerative pump with non-radial vanes," Bull of JSME, vol. 16, no. 98, pp. 1176-83, 1973.

[70] J. Burton, "The prediction and improvement of regenerative turbo-machine performance," BHRA, 1972.

[71] H. Abdallah, "A theoretical and experimental investigation of the regenerative pump with aerofoil blades," Ph.D. Thesis, Royal Military College of Science, UK, 1981.

[72] J. Song, M. Raheel and A. Engeda, "A compressible flow theory for regenerative compressors with aerofoil blades," Proc. IMechE Part, C: J. Mechanical Engineering Science, vol. 217, pp. 1241-57, 2003.

[73] K. Kusagaya, Y. Ito, M. Ito, Y. Mori, M. Takagi, K. Maruyama and E. Iwanari, "Impeller and turbine type fuel pump". USA Patent U.S. Patent 6767179, 27 Jul 2004.

[74] J. Kidger, R. Cumming and J. Gibbons, "Pumps". USA Patent U.S. Patent 5,435,692, 25 Jul 1995.

[75] A. Shirinov and S. Oberbeck, "High vacuum side channel pump working against atmosphere," Vacuum, vol. 85, no. 12, pp. 1174-7, 2011.

[76] M. Shimosaka and S. Yamazaki, "Research on the characteristics of regenerative pump," Bull of JSME, vol. 3, no. 10, pp. 185-90, 1960.

[77] S. Mason, "Influence of internal geometry upon regenerative pump performance," Senior Thesis, Massachusetts Institute of Technology, USA, 1957.

[78] H. Pfaff, "Comparative investigations on side channel pumps with and without inducer," Transl. from: Production Engineering Research Association, 1961.

[79] R. Bicard, "Investigation of the inlet design of the regenerative pump," Massachusetts Institiute of Technology, USA, 1957.

[80] H. Horiguchi, K. Wakiya, Y. Tsujimoto, M. Sakagami and S. Tanaka, "Study for the Increase of Micro Regenerative Pump Had," IJFMS, vol. 2, no. 3, pp. 189-95, 2009. 